\title{
Aspectos Históricos, Teóricos e Metodológicos da Teoria do Momentum Comportamental
}

\section{Historical, theoretical and methodological aspects of Behavioral Momentum Theory}

\section{Aspectos históricos, teóricos y metodológicos de la Teoría del Momentum Conductual}

\author{
André Luiz ${ }^{1}$, Carlos Eduardo Costa ${ }^{2}$, Carlos Renato Xavier Cançado ${ }^{3}$
}

[1] Universidade Estadual de Londrina/Núcleo Evoluir [2] Universidade Estadual de Londrina [3] Universidade de Brasilia I Título abreviado: Aspectos históricos, teóricos e metodológicos da Teoria do Momentum Comportamental I Endereço para correspondência: : Universidade Estadual de Londrina - Centro de Ciências Biológicas - Departamento de Psicologia Geral e Análise do Comportamento - Caixa Postal: 10.011, 86057-970, Londrina, PR I Email:andreluizbmt@gmail.com I doi: 10-18761/PAC.TAC.2019.007

Resumo: A Teoria do Momentum Comportamental (TMC) foi proposta para sistematizar os dados de pesquisas sobre resistência do comportamento à mudança, definindo-a como a tendência de um comportamento continuar ocorrendo quando se altera o ambiente no qual ele está sendo mantido. O presente artigo discorre acerca dos aspectos históricos, teóricos e metodológicos sobre a resistência do comportamento à mudança de acordo com o quadro conceitual da TMC e apresenta algumas das implicações dessa área de pesquisa para a Análise do Comportamento Aplicada. Observa-se que a resistência comportamental é um fenômeno complexo que pode ser afetado por diversas variáveis, tais como a taxa ou a magnitude dos reforços, diferentes exigências sobre a taxa de respostas e pelo atraso dos reforços. Tais resultados promoveram o desenvolvimento e refinamento de protocolos de intervenção, principalmente no trabalho com indivíduos com desenvolvimento atípico. A continuidade da pesquisa nessa área fornecerá maior e melhor conhecimento sobre o comportamento dos organismos, bases para revisões teóricas sobre os determinantes da resistência do comportamento à mudança bem como promoverá o desenvolvimento de intervenções comportamentais mais efetivas.

Palavras-chave: Persistência, resistência à mudança, artigo de revisão, momentum comportamental; ressurgência 
Abstract: Behavioral Momentum Theory (BMT) was proposed to systematize research data on behavioral resistance to change, defining it as the tendency of a behavior to continue occurring when the environment in which it is being maintained is altered. This article discusses the historical, theoretical and methodological aspects of behavioral resistance to change according to the conceptual framework of BMT and presents some of the implications of this area for Applied Behavior Analysis. It is noted that behavioral resistance is a complex phenomenon that can be affected by several variables, such as the rate or magnitude of reinforcement, different response-rate requirements and reinforcement delay. Such results promoted the development and refinement of intervention protocols, mainly in the work with individuals with atypical development. The continuity of research in this area will provide greater and better knowledge about the behavior of organisms, bases for theoretical reviews on the determinants of behavioral resistance to change, as well as promote the development of more effective behavioral interventions.

Keywords: Persistence, resistance to change, review article, behavioral momentum, resurgence

Resumen: La Teoría del Momentum Conductual (TMC) fue propuesta para sistematizar los datos de investigaciones sobre la resistencia de la conducta al cambio, definiéndolo como la tendencia de una conducta continuar ocurriendo cuando se altera el ambiente que la mantenía. El presente artículo discurre sobre los aspectos históricos, teóricos y metodológicos acerca de la resistencia de la conducta al cambio de acuerdo con lo marco conceptual de la TMC y presenta algunas de las implicaciones de esta área de investigación para el Análisis Conductual Aplicada. Se observa que la resistencia conductual es un fenómeno complejo que puede ser afectado por diversas variables, como la tasa o la magnitud de refuerzos, diferentes exigencias sobre la tasa de respuestas y el retraso de los refuerzos. Estos resultados promovieron la creación y el refinamiento de protocolos de intervención, principalmente en el trabajo con individuos con desarrollo atípico. La continuidad de investigaciones en esta área proporcionará más grande y mejor conocimiento sobre la conducta de los organismos, bases para revisiones teóricas sobre los determinantes de la resistencia de la conducta al cambio, así como promover el perfeccionamiento de intervenciones comportamentales más efectivas.

Palabras-clave: Persistencia, resistencia al cambio, artículo de revisión, momentum conductual, resurgimiento

\footnotetext{
Notas:

André Luiz - Professor Adjunto na Unopar - Arapongas. Pesquisador no Núcleo Evoluir Londrina. Mestre em Análise do Comportamento pela UEL. Foi bolsista CAPES/DS, durante a confecção deste artigo. Membro da REDETAC.

Carlos Eduardo Costa - Docente do Departamento de Psicologia Geral e Análise do Comportamento da UEL. Bolsista Produtividade em Pesquisa Produtividade em Pesquisa CNPq (PQ2, Processo: 311170/2016-1). Membro da REDETAC.

Carlos Renato Xavier Cançado - Professor e Pesquisador Visitante no Programa de PósGraduação em Ciências do Comportamento, Departamento de Processos Psicológicos Básicos, Instituto de Psicologia, Universidade de Brasília.
} 
Perguntas relacionadas a "como se tornar mais persistente" podem assombrar qualquer pessoa que precisa se manter exercendo uma ação continuamente, como estudar algo em que não se tem muito interesse por um determinado tempo sem se distrair ou se manter abstêmio após ter passado por um tratamento. Para os psicólogos clínicos, a persistência comportamental é tão importante que acaba por delimitar dois dos principais objetivos do processo terapêutico: (1) como tornar comportamentos prejudiciais menos persistentes? (2) como fortalecer comportamentos adequados para que eles persistam em face de eventuais mudanças que possam ocorrer como, por exemplo, o fim do processo terapêutico? (Nevin, 1996, 2015; Nevin \& Shahan, 2011).

Cumprir os dois objetivos expostos anteriormente exige dos profissionais amplo conhecimento acerca das variáveis que podem afetar a persistência comportamental ou, como mais comumente denominada, a resistência do comportamento à mudança. A maneira mais eficiente para conhecer essas variáveis é entrar em contato com estudos que investigaram esse fenômeno ou que sistematizaram informações sobre a área de estudo.

Um bom exemplo de sistematização de estudos que investigaram a resistência do comportamento à mudança é o capítulo de livro escrito por Santos (2005). De forma muito bem estruturada, Santos retrata algumas das variáveis que podem afetar a resistência do comportamento à mudança, quais são seus processos determinantes, descreve aproximações com os temas de Escolha e Preferência, Sensibilidade comportamental e História comportamental. Além disso, apresenta possíveis aplicações dos conceitos da Teoria do Momentum Comportamental (TMC). De maneira complementar ao capítulo de Santos, o presente artigo apresentará mais detalhadamente o processo histórico de desenvolvimento das investigações sobre resistência do comportamento à mudança, atualizará as referências de estudos voltados para a aplicação e discutirá possíveis relações com os estudos sobre ressurgência comportamental, cujo procedimento têm sido proposto como um modelo experimental de recaída (e.g., de comportamentos previamente mantidos por drogas ou outros comportamentos de relevância clínica e social). Conjuntamente, a estrutura do presente artigo foi pensada para que leitores com pouca familiaridade na área tenham maior facilidade para entender a TMC, as principais medidas e métodos empregados. Portanto, o primeiro assunto a ser abordado é o processo histórico de construção da área de estudo sobre resistência à mudança, iniciando pelas correlações entre os conceitos de "força da resposta" e "resistência comportamental".

\section{Força da resposta: da mecânica clássica ao comportamento dos organismos}

O fenômeno da resistência do comportamento à mudança tem sido estudado sob a ótica da Análise do Comportamento há mais de 50 anos (Craig, Nevin, \& Odum, 2014; Nevin, 1974; Nevin \& Grace, 2000; Nevin \& Wacker, 2013.). A TMC foi proposta para sistematizar os dados de pesquisas na área, definindo esse fenômeno como a tendência de um comportamento continuar ocorrendo quando se altera o ambiente no qual ele está sendo mantido (Nevin, 1974; ver também Cohen, 1998 e Craig et al., 2014). Portanto, quanto maior a mudança em um comportamento, dada alterações nas contingências que o mantinham, menor é sua resistência à mudança. Todavia, outras análises e definições já haviam sido utilizadas para descrever o quanto comportamentos podem mudar ou como avaliar mudanças comportamentais, tal como o conceito de "força da resposta".

Duas das definições de força da resposta de maior impacto para a Análise do Comportamento foram formuladas por Skinner (1938) e por Nevin (1974). Skinner, sugeriu que a força de uma resposta poderia ser definida por meio da observação do número de vezes que um organismo responde em uma determinada unidade de tempo (mas ver Nevin \& Grace, 2000 para outra noção de Skinner sobre esse conceito). Nesse sentido, uma taxa de respostas relativamente mais alta indicaria maior força do que uma taxa de respostas relativamente mais baixa.

Todavia, a taxa de respostas é uma dimensão condicionável do comportamento, o que dificulta seu uso para inferir a sua "força". Por exemplo, 
quando um esquema de reforçamento diferencial de baixas taxas de respostas (DRL) está em vigor, o responder tende a ocorrer "mais lentamente". Por outro lado, quando um esquema de reforçamento diferencial de altas taxas de respostas (DRH) está em operação o responder tende a ocorrer "mais rapidamente". Como as diferentes taxas de respostas resultam das contingências em vigor em cada esquema (i.e., DRL e DRH), seria impreciso afirmar que uma é mais (ou menos) forte do que a outra. Isto é, taxas de respostas podem ser mais altas ou mais baixas em função da contingência em vigor, mas igualmente "fortes".

Nevin (1974), por outro lado, sugeriu que a força da resposta deveria ser definida a partir do quanto um determinado desempenho muda ao se alterar o ambiente no qual ele está sendo mantido. Ou seja, a "força" de uma resposta é medida a partir do quanto um comportamento resiste as mudanças ambientais, independentemente da velocidade (i.e., da taxa) com que a resposta ocorre.

Para que a resistência à mudança pudesse ser medida, a partir da noção proposta por Nevin (1974), seria necessário o estabelecimento de uma taxa de respostas relativamente estável e que alguma modificação nas contingências que mantinham o responder fosse realizada (e.g., saciação, extinção, punição etc.). A esse tipo de alteração nas contingências, atribui-se o nome de "operação perturbadora" [do inglês, disrupting operation - DO]. Em 1983, Nevin, Mandell e Atak propuseram que a resistência à mudança frente a operações perturbadoras poderia ser entendida por meio de uma analogia com a Segunda Lei de Newton. É importante destacar que a proposta dessa analogia foi, possivelmente, muito influenciada por Nevin ser formado em engenharia naval, o que levou ao desenvolvimento de muitas formulações matemáticas para descrever e prever o comportamento dos organismos (Nevin, 2015).

De acordo com a Segunda Lei de Newton, qualquer alteração da velocidade de um corpo em movimento é diretamente proporcional à for- ça imposta sobre ele e inversamente proporcional a sua massa (Nevin, 2015; Nevin \& Wacker, 2013). Em um laboratório experimental de Física, por exemplo, pesquisadores poderiam testar a Segunda Lei de Newton utilizando um vagão de trem em miniatura e um delineamento experimental entre fases, ou seja, com a exposição de um corpo a duas ou mais condições espaçadas temporalmente. Levando em consideração que todas as variáveis são controladas (e.g., atrito nos trilhos, massa do trem, velocidade, temperatura etc.), um mecanismo é acionado para que ele dê início ao movimento do vagão do trem. Após o vagão atingir uma velocidade constante, aplica-se uma força contra ele (e.g., aumento no atrito do trilho, devidamente controlado) e avalia-se qual foi a alteração em sua velocidade. Posteriormente, os pesquisadores dobram a massa do vagão e realizam o mesmo procedimento, com o mesmo controle experimental, e avaliam novamente os efeitos sobre a mudança na velocidade do vagão do trem comparando com os efeitos do teste anterior. De acordo com a Segunda Lei de Newton, a velocidade do vagão deveria mudar (desacelerar) mais rapidamente no primeiro do que no segundo teste, quando sua massa foi dobrada.

A analogia com a Segunda Lei de Newton proposta por Nevin et al. (1983) deu origem ao termo Momentum Comportamental, que se refere ao produto da taxa de respostas e a resistência à mudança (Santos, 2005). A Figura 1 descreve, à esquerda, a Segunda Lei de Newton, e à direita a analogia comportamental dessa lei utilizada na TMC.

A tradução comportamental da Segunda Lei de Newton foi e ainda é utilizada em diversas investigações sobre a resistência do comportamento à mudança. Contudo, como apontado por Nevin e Grace (2000), a analogia com a física clássica newtoniana não é nada mais que um recurso verbal útil para descrever a relação entre a resistência do comportamento à mudança e variáveis ambientais que alteram essa resistência. 

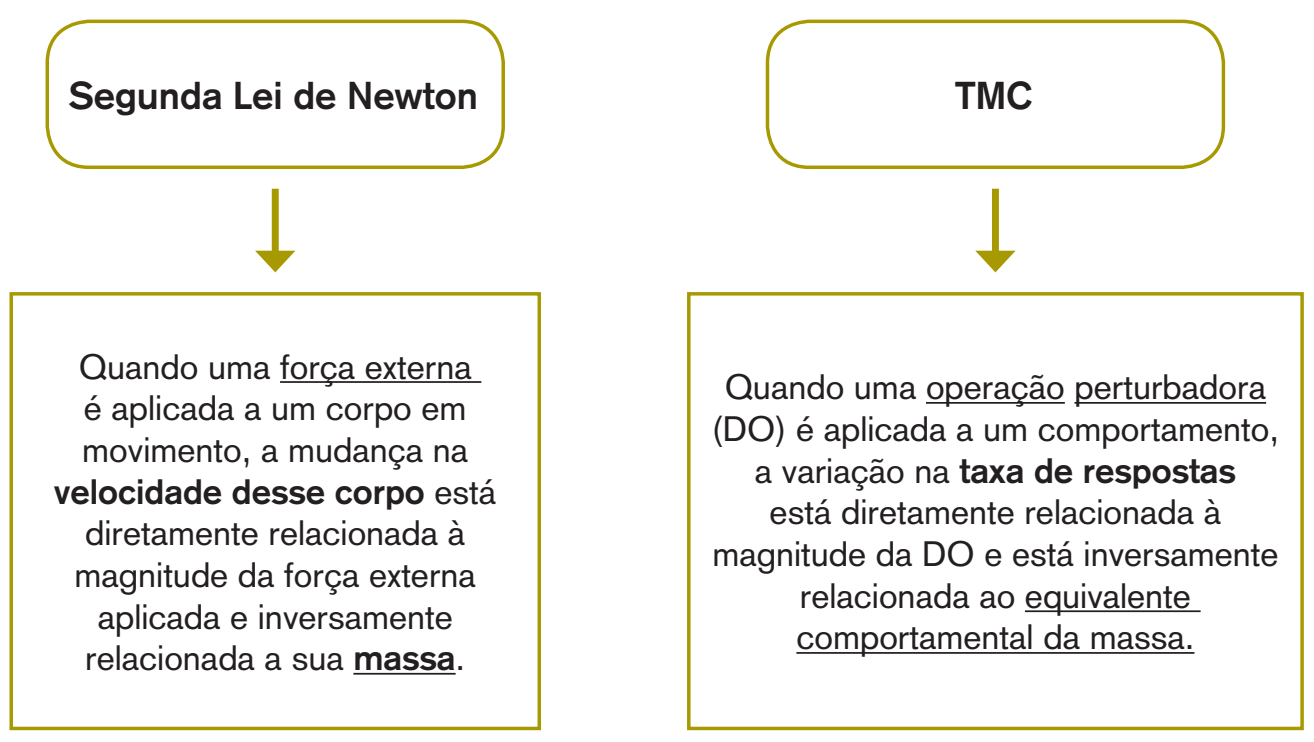

Figura 1. Segunda Lei de Newton (à esquerda) e sua analogia na TMC (à direita)

\section{Em busca do controle experimental}

O controle experimental em um laboratório de Física pode ser muito mais refinado do que o controle experimental exercido no estudo do comportamento dos organismos. Isso ocorre, por exemplo, pela própria diferença entre objetos de estudo e pela possiblidade limitada do controle de variáveis presentes na história extra laboratorial de cada organismo. Nesse sentido, a utilização de um delineamento experimental entre fases para o estudo da resistência à mudança (como no exemplo acima do experimento em um laboratório de Física), ainda que possível, pode ter seus resultados prejudicados por conta de variações ambientais que possam ocorrer fora do laboratório ou mesmo pelos efeitos de história comportamental que se acumulam ao longo das exposições de um organismo às condições experimentais (cf. Costa, Cirino, Cançado, \& Soares, 2009). Por exemplo, em um primeiro momento, treina-se um rato para pressionar a barra sob um esquema de razão fixa (FR), no qual alimento será produzido após um determinado número fixo de respostas. Após estabilizada a taxa de respostas, altera-se o esquema para Extinção (EXT). Em um segundo momento, o mesmo rato é exposto a outro esquema de reforçamento e, após estabilizada a taxa de respostas, altera-se o esquema para EXT. É muito provável que a taxa de respostas diminua mais rapidamente na segunda exposição à EXT do que na primeira, possivelmente por conta da história de exposição à EXT e não porque um esquema de reforçamento tenha produzido um desempenho mais resistente à EXT do que outro (Anger \& Anger, 1976; Baum, 2012).

Uma possível solução para essa limitação seria o emprego de delineamentos de grupo. Em um mesmo dia, os sujeitos ou participantes de dois ou mais grupos passam por duas ou mais situações diferentes e os desempenhos comportamentais de cada grupo são comparados. Contudo, os efeitos das variáveis manipuladas podem ser distintos em cada grupo e, ainda que muitos modelos estatísticos sejam utilizados para avaliar as diferenças de desempenho dos sujeitos que compõem um grupo, esse tipo de delineamento não reflete com clareza as mudanças comportamentais ocorridas no comportamento de cada organismo (ver Sidman, 1960/1976 para outras diferenças dos delineamentos de grupo e intrassujeitos).

Dadas as limitações de delineamentos entre fases e de delineamentos de grupo expostas acima, Nevin (1974) propôs que a resistência do comportamento à mudança fosse estudada, primariamente, por meio delineamentos experimentais intrassujeitos, em que se compara o desempenho de um mesmo organismo antes e depois das modificações ambientais (Sidman, 1960/1976; 
Sampaio, Azevedo, Cardoso, Lima, Pereira \& Andery, 2008) e por meio do emprego de esquemas de reforçamento múltiplo, cuja a estrutura é ilustrada na Figura 2

Como pode ser visto na Figura 2, em um esquema de reforçamento múltiplo, dois ou mais estímulos exteroceptivos (e.g., sons, luzes etc.) diferentes são correlacionados com dois ou mais esquemas de reforçamento (e.g., VI 10 s e VI 30 s). Isso possibilita que os diferentes esquemas (i.e., os componentes do esquema múltiplo) ocorram de forma alternada em uma mesma sessão experimental. Assim, pode-se comparar o desempenho do mesmo sujeito, em cada componente do esquema múltiplo, antes e depois da manipulação de uma variável (i.e., intrassujeitos, intra e entre sessões).

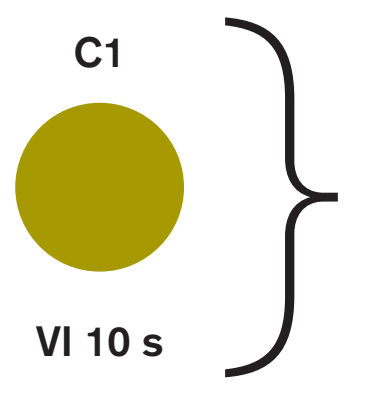

Na presença da luz apagada, consequências são liberadas em média a cada $10 \mathrm{~s}$ para a resposta que cumprir a contingência

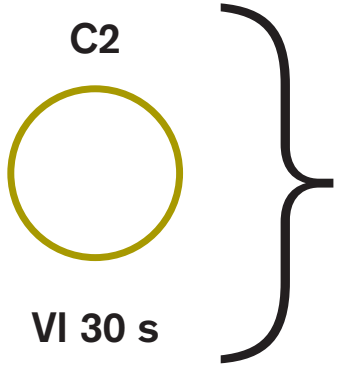

Na presença da luz acesa, consequências são liberadas em média a cada 30s para a resposta que cumprir a contingência

Figura 2. llustração de um esquema múltiplo intervalo variável (VI) VI. Na presença da luz apagada (C1) consequências são produzidas pela primeira resposta após um intervalo médio de $10 \mathrm{~s} \mathrm{e,} \mathrm{na} \mathrm{presença} \mathrm{de} \mathrm{luz} \mathrm{acesa} \mathrm{(C2),} \mathrm{consequências} \mathrm{são}$ produzidas pela primeira resposta após um intervalo médio de $30 \mathrm{~s}$.

Refinamentos experimentais e a adoção da proporção de mudança (PM) como medida da resistência à mudança

A partir da proposta de Nevin (1974), o procedimento frequentemente utilizado para investigar a resistência do comportamento à mudança ocorre da seguinte maneira: primeiramente, um organismo é exposto a um esquema múltiplo, durante a linha de base (LB), na qual dois ou mais operantes são selecionados e a variável experimental de interesse é manipulada entre os componentes do esquema múltiplo em vigor de acordo com um delineamento intrassujeito. Em seguida, inicia-se o teste em que os estímulos aos quais os componentes estavam correlacionados são mantidos e DOs, tais como saciação (e.g., Aló, Abreu-Rodrigues, Souza, \& Cançado, 2015; Podlesnik \& Shahan, 2008), EXT (e.g., Nevin, 1974, Experimento 2; Nevin, Tota, Torquato, \& Shull, 1990), comida independente da resposta no intervalo entre componentes do esquema múltiplo (e.g., Lattal, 1989; Nevin, 1974,
Experimento 1), perda de pontos (Ponce, 2014) etc., são aplicadas (ver Aló \& Costa, no prelo, para uma descrição mais detalhada do arranjo experimental).

Uma vez que um organismo passou pelas condições descritas acima, compara-se a taxa de respostas em cada componente do esquema múltiplo durante o Teste com a taxa de respostas em cada componente durante a LB (usualmente considera-se a média da taxa de respostas nas últimas sessões da LB como representativa do estado estável do comportamento). Isto é feito calculando-se, para cada componente, a taxa de respostas no Teste como proporção da taxa de respostas na LB (uma medida que chamaremos nesse artigo de proporção de mudança, PM). Portanto, PM = taxa de respostas no Teste / taxa de respostas na LB.

Após calcular a PM é possível verificar se houve mudança na taxa de respostas no Teste em relação à LB para cada componente e se houve mudança diferencial entre os componentes. 
Considere o exemplo hipotético a seguir: um rato foi exposto a um esquema múltiplo VI 30 s VI 90 s durante a LB. No VI 30 s (C1), havia uma luz piscando e o rato emitiu em média 120 respostas por minuto (R/min). No VI $90 \mathrm{~s}(\mathrm{C} 2)$, havia uma luz acesa continuamente e o rato emitiu em média $60 \mathrm{R} / \mathrm{min}$. O Teste foi composto por um esquema múltiplo EXT EXT em que os estímulos (luzes) correlacionados aos componentes na LB foram mantidos, mas as respostas não produziam mais consequências. No Teste, a taxa de respostas no $\mathrm{C} 1$ diminuiu para $80 \mathrm{R} / \mathrm{min}$ e no $\mathrm{C} 2$ para $20 \mathrm{R} /$ min. Portanto, em ambos os componentes, a taxa de respostas diminuiu $40 \mathrm{R} / \mathrm{min}$.

Inicialmente, poder-se-ia dizer que não houve resistência à mudança diferencial entre os componentes descritos anteriormente, já que a redução na taxa de respostas foi semelhante entre eles. Porém, ao realizar o cálculo de PM é possível medir qual foi a mudança relativa em cada componente. Como visto anteriormente, para $\mathrm{C} 1$, divide-se a taxa de respostas no Teste pela taxa de respostas na LB (i.e., 80/120) obtendo-se 0,6 (i.e., no Teste, a taxa de respostas ocorre com um valor que é $60 \%$ do que ocorria na LB; alternativamente, pode-se dizer que a taxa de respostas nesse componente reduziu $40 \%$ em relação à LB). Para C2, faz-se o mesmo cálculo (i.e., 20/60) obtendo-se 0,3 (i.e., no Teste, a taxa de respostas ocorre com um valor que é $30 \%$ do que ocorria na LB; alternativamente, pode-se dizer que a taxa de respostas nesse componente reduziu 70\% em relação à LB). Se não houvesse mudança na taxa respostas no Teste em relação a $\mathrm{LB}$ o valor da $\mathrm{PM}$ deveria ser 1,0 . Sendo assim, quanto mais próximo de 1,0 mais o comportamento foi resistente à mudança. Dessa forma, o comportamento no $\mathrm{C} 1$ $(\mathrm{PM}=0,6)$ foi mais resistente à mudança do que o comportamento no $\mathrm{C} 2$ ( $\mathrm{PM}=0,3$; ver Aló \& Costa, no prelo, para outros exemplos de descrição da PM; ver também Craig et al., 2014).

Além da importância da medida de PM nos estudos sobre resistência à mudança, observa-se no exemplo anterior que o esquema de reforçamento escolhido foi o VI. Esquemas VI são muito úteis quando se busca estabelecer taxas de respostas relativamente constantes. Além disso, variações na taxa de respostas não afetam substancialmente a taxa de reforços. Outra característica importante do arran- jo experimental ilustrado na Figura 2 é a programação de um intervalo entre os componentes (IEC). O IEC consiste em um período em que os estímulos correlacionados a cada componente não são apresentados (tomando o exemplo descrito na Figura 2, a luz poderia ficar apagada e soar um som por $30 \mathrm{~s}$ após a apresentação de cada componente do esquema múltiplo VI 10 s VI 30 s) e ajuda a assegurar que os componentes representem situações de estímulos relativamente distintas (i.e., diminui a probabilidade interação entre as contingências em vigor em ambos os componentes; cf. Reynolds, 1961). Com isso, o mesmo evento perturbador pode ser aplicado a várias situações de estímulos na mesma sessão, eliminando potenciais efeitos de variáveis estranhas (Craig et al., 2014; Nevin, 2015).

Até agora foram apresentados aspectos históricos e metodológicos das investigações sobre resistência do comportamento à mudança e da TMC. Essa revisão provavelmente ajudará o leitor a entender os procedimentos e medidas encontrados na bibliografia experimental sobre o tema.

\section{Uma breve revisão da bibliografia experimental}

Nevin (1974, Experimento 1) ${ }^{1}$ treinou pombos para bicarem um disco sob um esquema múltiplo VI 60 s VI 180 s durante a LB. No Teste, foi liberada comida independente da resposta durante o IEC. Os resultados indicaram que o comportamento mantido pelo VI $60 \mathrm{~s}$ (que liberava 60 reforços por hora) foi mais resistente à mudança do que o comportamento mantido pelo VI $180 \mathrm{~s}$ (que liberava 20 reforços por hora). Resultados semelhantes foram replicados com ratos (Cohen, 1998) e peixes (Igaki \& Sakagami, 2004) como sujeitos experimentais.

No estudo de Mace et al. (1990, Parte 1), humanos com desenvolvimento atípico foram expostos a um esquema múltiplo VI 60 s VI 240 s e a tarefa experimental consistiu na armazenagem de dois grupos de objetos plásticos com cores diferentes dentro de um pote. As cores dos objetos caracterizavam

1 O capítulo de Aló e Costa, no prelo, descreve os cinco experimentos do artigo de Nevin (1974), de maneira mais detalhada. 
os diferentes estímulos a que os componentes do esquema múltiplo foram correlacionados. No Teste, a apresentação de um vídeo concomitante à realização da tarefa experimental foi utilizada como DO. A resistência à mudança foi maior no componente correlacionado ao VI 60 s (i.e., à maior taxa de reforços). Resultados semelhantes foram encontrados com humanos adultos com desenvolvimento típico por Cohen (1996, Experimento 1) e por Kuroda, Cançado e Podlesnik (2016).

Os resultados de experimentos descritos até o momento demonstraram que a resistência do comportamento à mudança parece ser função positiva da taxa de reforços (i.e., quanto maior a taxa de reforços na presença de um determinado estímulo, maior é a resistência do comportamento à mudança na presença desse estímulo quando operações perturbadoras estão em vigor). Porém, o que aconteceria se reforços independentes da resposta fossem adicionados em um dos componentes do esquema múltiplo? Essa foi uma das questões que deu início a um novo ciclo de investigações sobre as variáveis envolvidas nos estudos sobre resistência à mudança.

Nevin et al. (1990) expuseram pombos a um esquema múltiplo com dois componentes na LB. Em ambos os componentes, bicadas no disco de resposta produziam alimento de acordo com um VI 60 s. Além disso, em um dos componentes, o alimento também foi liberado independentemente da resposta de acordo com um esquema de tempo variável (VT) $30 \mathrm{~s}$ sobreposto ao VI $60 \mathrm{~s}$. Isso permitiu que o número de reforços liberados de maneira dependente da resposta fosse o mesmo entre os componentes, mas que em um deles houvesse maior quantidade de reforços obtidos (i.e., 60 reforços por hora, dependentes da resposta, em um componente e 60 reforços por hora, dependentes da resposta, mais 120 reforços por hora, independentes das respostas, no outro componente). No Teste, estava em vigor um esquema múltiplo EXT EXT. Os resultados obtidos podem ser divididos em duas partes: (1) a taxa de repostas durante a LB foi menor no componente com a adição de alimento em VT; (2) a resistência do comportamento à mudança foi maior no componente com adição de alimento em VT. Esses resultados demonstraram que o quanto um organismo responde (taxa de res- postas) e o quanto esse responder resiste à mudança são aspectos independentes do comportamento. Essa independência da taxa de respostas e da resistência à mudança será discutida mais adiante. Resultados semelhantes foram obtidos por Grimes e Shull (2001) e Shahan e Burke (2004) utilizando ratos como sujeitos e arranjando consequências independentes da resposta qualitativamente diferentes das dependentes da resposta durante a LB (e.g., leite condensado e comida, no estudo de Grimes \& Shull, 2001 e etanol e comida, no estudo de Shahan \& Burke, 2004).

Mace et al., (1990, Parte 2), replicaram o estudo de Nevin et al., (1990) com humanos com desenvolvimento atípico. A tarefa experimental, assim como a operação perturbadora, foi a mesma realizada na Parte 1 desse estudo, descrita anteriormente. Em ambos os componentes do esquema múltiplo, reforços foram liberados contingentes à tarefa experimental em um esquema VI 60 s. Além disso, em um dos componentes, reforços foram liberados sob um VT 30 s (i.e., reforços independentes da resposta). A taxa de respostas foi menor, e a resistência à mudança foi maior, no componente do múltiplo em que reforços independentes adicionais estavam em vigor (i.e., VI + VT).

Os resultados obtidos por Mace et al., (1990, Parte 2), Nevin et al., (1990), Grimes e Shull (2001) e Shahan e Burke (2004) são contrários a noção de que taxas de respostas mais altas são mais "fortes" do que taxas de respostas mais baixas, simplesmente por serem mais altas. Por outro lado, parecem fortalecer o que é proposto pela analogia comportamental da Segunda Lei de Newton, sugerindo que a resistência do comportamento à mudança parece ser função da quantidade de reforços obtidos em um dado contexto (i.e., o equivalente comportamental da massa) e relativamente independente da taxa de respostas na LB (Mace et al., 1990; Partes 1 e 2; Nevin et al., 1990).

Relação semelhante à obtida entre a taxa de reforços e a resistência à mudança foi encontrada quando se manipulou a magnitude dos reforços entre componentes do esquema múltiplo na LB. Nevin (1974, Experimento 3), por exemplo, expôs pombos a um esquema múltiplo VI 60 s VI 60 s na LB (note que a taxa de reforços é a mesma em cada componente). Em um dos componentes, a duração do acesso ao 
alimento produzido pela resposta era de $2,5 \mathrm{~s}$ (i.e., menor magnitude). No outro componente, o tempo de acesso ao alimento foi de 7,5 s (i.e., maior magnitude). No Teste, alimento independente da resposta foi liberado durante o IEC. O comportamento foi mais resistente à mudança no componente correlacionado a maior magnitude dos reforços. Esses resultados foram replicados nos estudos de Harper e Mclean (1992, Experimento 1) e de Harper (1996), ambos com pombos como sujeitos experimentais.

Os resultados obtidos pelos estudos descritos até o momento sugerem que a resistência à mudança é função direta da taxa e da magnitude dos reforços (i.e., do total de reforços em um dado contexto) e não da taxa de respostas produzida em cada componente do múltiplo na LB. Com base nesses resultados, a TMC propõe que a resistência do comportamento à mudança e a taxa de respostas são dois aspectos independentes do comportamento operante (Craig et al., 2014; Nevin, 2015; Nevin et al., 1990; Nevin \& Wacker, 2013). Isto é, a resistência à mudança seria função da relação entre os estímulos antecedentes e os estímulos consequentes obtidos neste contexto (i.e., relação S-S), sejam eles obtidos de maneira dependente ou independente da resposta, qualitativamente semelhantes ou não. A taxa de respostas, por sua vez, seria função da relação entre o responder e os estímulos consequentes (i.e., relação R-S).

Se as variáveis envolvidas na relação S-S (i.e., taxa e magnitude dos reforços) fossem as únicas variáveis que determinam a resistência do comportamento à mudança, como proposto pela TMC, não deveria haver resistência à mudança diferencial quando essa relação fosse igualada entre os componentes de um esquema múltiplo de reforçamento. Contudo, tem-se demonstrado que quando a relação S-S é igualada entre os componentes, outras variáveis passam a controlar a resistência do comportamento à mudança, $\mathrm{o}$ que aponta um limite à proposição central da TMC de independência entre o quanto um organismo responde e o quanto esse responder resiste à mudança.

\section{Limites da Teoria}

No estudo de Grace, Schwendiman e Nevin (1998, Fase 2), a relação S-S foi igualada entre os componentes do esquema múltiplo de reforçamento e a variável manipulada foi o atraso dos reforços. $\mathrm{Na}$ LB, pombos foram submetidos a um esquema múltiplo com um VI $40 \mathrm{~s}$ em um componente e com um tandem VI $37 \mathrm{~s}$ tempo fixo (FT) 3 s no outro componente. No VI 40 s, após a ocorrência de uma resposta que cumprisse a contingência, o reforço era liberado de forma imediata. No tandem VI 37 s FT 3 s, a resposta deveria cumprir a contingência imposta pelo VI e, após isso, atrasava-se a liberação do reforço em 3 s. Note que a taxa de reforços foi semelhante entre os componentes do esquema múltiplo (i.e., um reforço foi liberado em média a cada 40 s). No Teste, esteve em vigor um esquema múltiplo EXT EXT. De forma contraditória à proposta central da TMC, foi observada maior resistência à EXT no componente correlacionado com o reforço imediato na LB.

Em outros estudos, a taxa e a magnitude dos reforços também foi igualada entre os componentes de um esquema múltiplo, mas manipulou-se contingências que exigiam taxas de respostas diferenciais na LB. Nevin (1974, Experimento 5), por exemplo, expôs pombos a um esquema múltiplo com dois componentes na LB. Em um componente, esteve em vigor um esquema tandem VI DRL que exigia que o responder ocorresse de forma relativamente mais "lenta" para que houvesse reforço. No outro componente, esteve em vigor um esquema tandem VI DRH que exigia que o responder ocorresse de maneira relativamente mais "rápida" para que houvesse reforço. No Teste esteve em vigor um esquema múltiplo EXT EXT ou liberação de reforços independentes da resposta durante o IEC. Sob relações S-S semelhantes, houve maior resistência à mudança no componente correlacionado ao tandem VI DRL do que ao tandem VI DRH. Resultados semelhantes foram obtidos por Lattal (1989) e Aló et al. (2015, Experimentos 1 e 2).

A partir dos resultados descritos anteriormente, passou-se a especular que taxas de respostas mais baixas na LB são mais resistentes à mudança durante o Teste do que taxas de respostas mais altas na LB, quando a relação S-S é igualada entre os componentes. Contudo, note que no estudo de Grace et al. (1998) em que foi manipulado o atraso do reforço, menor resistência à mudança foi correlacionada com a menor taxa de respostas na $\mathrm{LB}$, sugerindo que o esquema utilizado para estabelecer altas e 
baixas taxas de resposta a LB pode ser um determinante importante da resistência à mudança diferencial. Além disso, Aló et al. (2015, Experimento 3) demonstraram que essa relação pode também depender da operação perturbadora utilizada.

Aló et al. (2015, Experimento 3) também manipularam contingências que exigiam taxas de respostas diferenciais na LB (i.e., múltiplo FR DRL), mas obtiveram resultados diferentes dos demais estudos que realizaram essa manipulação ao utilizarem um esquema múltiplo intervalo fixo (FI) FI em conjunto com saciação como operação perturbadora durante o Teste. Os resultados obtidos sugeriram que o comportamento mantido na LB pelo componente correlacionado ao FR foi mais resistente à mudança do que o comportamento mantido na LB pelo componente correlacionado ao DRL. Aló et al. propuseram que a relação entre as contingências em vigor no teste e na LB também pode afetar a resistência do comportamento à mudança. No caso específico, a maior similaridade do intervalo entre reforços (IRI) entre um esquema FI com um FR, quando comparado com um DRL, pode ter sido responsável pelos resultados. Assim, pode ser complicado afirmar que quando a taxa e a magnitude dos reforços são igualadas entre os componentes de um esquema múltiplo, condições que produzem taxas mais baixas de respostas produzem também maior resistência à mudança. É necessário saber quais as condições na LB que produziram tais diferenças na taxa de respostas entre os componentes do esquema múltiplo, bem como quais operações perturbadoras estiveram em vigor no Teste.

Até o momento, observa-se que o pressuposto central da TMC de que a resistência do comportamento à mudança é função primária da relação S-S e independente da relação resposta-reforço necessita de maior sistematização (ver, Nevin et al., 2017). Independentemente de limites a esse pressuposto central da TMC, a maioria dos estudos têm demonstrado que a resistência do comportamento à mudança é uma função direta da taxa e da magnitude dos reforços. Esses resultados têm impulsionado novos tipos de intervenção comportamental.

\section{Do laboratório à aplicação e relações com a ressurgência comportamental}

Os resultados das inúmeras pesquisas básicas desenvolvidas para investigar o fenômeno da resistência do comportamento à mudança, de acordo com o quadro conceitual da TMC, sugerem que o comportamento previamente reforçado tenderá a continuar ocorrendo até que operações perturbadoras afetem o responder (Mace \& Nevin, 2017). Em ambientes aplicados, operações perturbadoras podem ser o encerramento da psicoterapia (tendo em vista o quanto os comportamentos adequados resistirão dado o término desse processo), o início da intervenção (avaliando a resistência à mudança de comportamentos inadequados), distratores (quando se está planejando contingências para ensinar um cliente a estudar melhor), entre outros. Com base nos resultados obtidos na pesquisa básica, pesquisas aplicadas foram desenvolvidas para criar e refinar intervenções comportamentais.

Mace et al. (1988), por exemplo, delinearam um procedimento para tratar comportamentos de desobediência (do inglês noncompliance) apresentados por humanos com desenvolvimento atípico. $\mathrm{O}$ procedimento utilizado, denominado como high- $p$ procedure, visava aumentar a taxa de reforços contingente a classe de comportamentos de obediência (do inglês compliance) e verificar os efeitos disso sobre a probabilidade de obediência às instruções que, geralmente, não eram seguidas. Para isso, foram selecionados dois tipos de instruções para serem dadas aos participantes: comandos com alta probabilidade de obediência (CAO) e comandos com baixa probabilidade de obediência (CBO). $\mathrm{Na}$ LB os participantes foram expostos a uma série de CBOs e avaliava-se a porcentagem de vezes que eram obedecidos. No Teste, cada CBO era precedido por uma sequência de três ou quatro CAO. Elogios descritivos (e.g., "Muito bem! Obrigado por ter guardado seu brinquedo") fornecidos pelos experimentadores e contingentes a realização das tarefas relacionadas aos comandos foram utilizados como reforços. Os resultados obtidos indicaram que o número de atividades cumpridas relacionadas aos $\mathrm{CBO}$ aumentou quando foram precedidas 
pelas sequências de CAO. Mace et al., sugeriram que ao expor os participantes a sequências de CAO, foi possível aumentar a taxa de reforços contingente a classe de comportamentos de obediência tornando-a mais provável de ocorrer na presença de possíveis eventos perturbadores, tais como os CBO. A principal implicação para a aplicação é que, planejar intervenções que aumentem a taxa de reforços para uma resposta específica (utilizando instruções com alta probabilidade de serem seguidas e, portanto, reforçadas), pode aumentar a probabilidade de obediência de instruções com baixa probabilidade de serem seguidas. Todavia, o procedimento $h i-$ $g h-p$ não é o único utilizado para o estabelecimento de comportamentos desejáveis.

Em processos terapêuticos, é comum a utilização de esquema de reforçamento para diminuir a ocorrência de respostas indesejáveis ao mesmo tempo em que se estabelece e fortalece respostas desejáveis. Mace et al. (2010) realizaram três experimentos para avaliar os efeitos da utilização de um esquema DRA sobre a resistência do comportamento à mudança. No Experimento 1 (ver figura 3), indivíduos com desenvolvimento atípico foram expostos a um arranjo ambiental com duas condições na LB. Na Condição 1, reforços foram liberados somente quando uma resposta considerada indesejável (i.e., resposta alvo - RALV) era apresentada. $\mathrm{Na}$ Condição 2, respostas alternativas (RALT) foram reforçadas de maneira concorrente a RALV, no mesmo contexto (como comumente ocorre quando um DRA é planejado como intervenção). No Teste, sessões de EXT estiveram em vigor. Apesar da taxa de respostas do comportamento indesejável (RALV) ter diminuído quando DRA foi implementado, a resistência à extinção da RALV foi maior após a Condição 2 (com DRA) do que após a Condição 1 (sem DRA). Portanto, os resultados demonstraram que há diminuição na ocorrência de respostas indesejáveis (i.e., RALV) ao utilizar um esquema DRA, porém como os reforços no DRA ocorrem no mesmo contexto em que ocorrem os reforços para o comportamento RALV, há um fortalecimento da relação S-S e consequentemente maior resistência à mudança (resistência à extinção no experimento descrito).

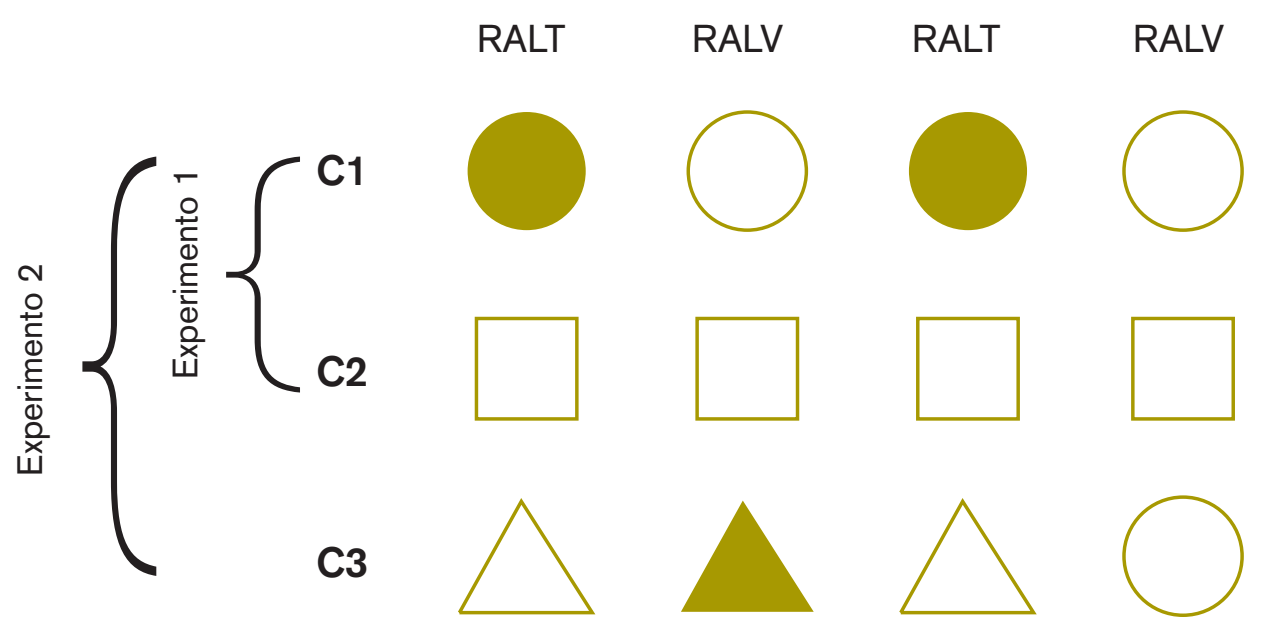

Figura 3. Diagrama dos estímulos discriminativos e condições (C) empregadas nos Experimentos 1 e 2 de Mace et al. (2010) durante a LB (painel à esquerda) e durante o Teste (painel à direita). Estímulos abertos na LB representam o componente no qual havia reforços. Estímulos preenchidos indicam o componente no qual não havia reforços. Quando ambos os estímulos estão abertos havia reforçamento concorrente (DRA). No Teste, os estímulos foram idênticos aos da LB, mas foi empregada extinção (EXT) nas três condições. Durante o Teste, C3 foi composta pelos estímulos em que, na LB, havia reforços somente para RALT e somente para RALV. 
No Experimento 2, Mace et al. (2010) utilizaram quatro ratos como sujeitos experimentais e, aplicando um procedimento semelhante ao do Experimento 1, identificaram que quando a liberação de reforços para a RALT ocorria na presença de um estímulo antecedente diferente daquele em que foram liberados reforços para a RALV, a resistência à mudança da RALV, em sessões de EXT, foi menor do que quando o reforçamento para RALT e para a RALV ocorria na presença do mesmo estímulo antecedente. A partir dos resultados do Experimento 2, Mace et al., retomaram as investigações com humanos no Experimento 3 e, diferindo o estímulo antecedente quando havia reforçamento concorrente para a RALT e para a RALV, foram obtidos resultados semelhantes aos do Experimento 2. Os autores concluíram que os efeitos do aumento da resistência do comportamento à mudança causados pela utilização de DRA podem ser reduzidos ao se arranjar estímulos antecedentes diferentes daqueles correlacionados com a história de reforçamento de respostas indesejáveis (ver também, Podlesnik, Bai, \& Elliffe, 2012).

Além do DRA, o procedimento de reforçamento não contingente (RNC), que consiste na liberação de reforços independente da resposta (Vollmer, Iwata, Zarcone, Smith, \& Mazaleski, 1993), também é amplamente utilizado para redução da ocorrência de comportamentos destrutivos apresentados por indivíduos com desenvolvimento atípico. Saini, Fisher, Maegan e Pisman (2017) avaliaram a resistência do comportamento à mudança após um procedimento de RNC empregado com ou sem EXT. Foram duas as condições principais: (1) RNC (VT 30 s) com EXT do comportamento alvo (análogo ao comportamento destrutivo); (2) RNC (VT $30 \mathrm{~s}$ ) e o comportamento alvo produzia reforços em VI 30 s. EXT foi empregada durante o Teste. Maior resistência à mudança ocorreu para o comportamento alvo após a Condição 2, na qual a relação $S-S$ foi fortalecida pela soma dos reforços em VI e VT.

Os resultados obtidos nos estudos que empregaram DRA e RNC demonstraram o quanto a relação S-S é importante na estruturação de planos de intervenção. Boas intervenções vão além do conhecimento acerca da história de vida do cliente ou sobre quais são as contingências mantenedoras do comportamento problema. Deve-se elaborar um plano de intervenção que leve em consideração os possíveis efeitos das variáveis manipuladas sobre a resistência à mudança do comportamento problema e dos comportamentos alternativos em face de eventuais mudanças (e.g., início de intervenções mais incisivas ou férias do cliente e/ou terapeuta).

Os dados obtidos pelos estudos de Mace et al. (2010), Podlesnik et al. (2012) e Saini et al. (2017) apresentaram uma importante implicação para o planejamento de intervenções: deve-se evitar adicionar outras fontes de reforços para comportamentos alternativos no mesmo contexto em que o comportamento problema é reforçado. Todavia, esses estudos avaliaram somente comportamentos mantidos por consequências extrínsecas, mas existem outros comportamentos mantidos por reforçamento automático, especialmente em indivíduos com desenvolvimento atípico.

Também utilizando o quadro conceitual da TMC, Ahearn, Clark, Gardenier, Chung e Dube (2003) investigaram os efeitos do fortalecimento da relação $S$-S sobre a resistência à mudança de comportamentos estereotipados, mantidos por reforçamento automático, em três crianças diagnosticadas com transtorno do espectro autista. $\mathrm{O}$ estudo foi composto por três fases: LB, Fase B e Teste. Durante a LB, apenas era medida taxa de respostas estereotipadas (reforçadas automaticamente). Na Fase B, os participantes tinham acesso a itens com alto grau de preferência disponibilizados em VT e as respostas estereotipadas poderiam continuar ocorrendo. A introdução dos itens em VT diminuiu a taxa de respostas estereotipadas. Todavia, note que na Fase $B$, em comparação à LB, a relação S-S foi fortalecida pela soma de reforços em VT (acesso aos itens) com os reforços automáticos das respostas estereotipadas. No Teste, os itens com alto grau de preferência foram disponibilizados continuamente. As três fases foram apresentadas em duas sequências: (1) LB, Fase B, Teste e LB; (2) LB, LB, Teste e LB. A Sequência 2 possuía duas LB seguidas para que a taxa de reforço (automático) para o comportamento estereotipado tivesse mais possibilidade de ocorrência. Os resultados indicaram que as respostas estereotipadas foram mais resistentes à mudança na Sequência 1 em que o Teste ocorria após a Fase B, ou seja, quando a relação S-S era fortalecida, do 
que na Sequência 2 em que o Teste ocorria após a LB (sem adição de reforços em VT).

Além dos reforços automáticos, outra relação de grande importância no ambiente clínico, por exemplo, são os reforços incondicionados. Vargo e Ringdahl (2015) investigaram e compararam a resistência à mudança de comportamentos mantidos por reforços condicionados e incondicionados em quatro experimentos, com crianças com desenvolvimento típico. No Experimento 1, identificaram-se reforçadores condicionados e incondicionados de igual preferência. Durante a LB dos Experimentos 2, 3 e 4 os participantes foram expostos a um esquema múltiplo VI $30 \mathrm{~s}$ VI 30 s e o Teste consistiu em EXT, atividade concorrente e comida prévia (Experimentos 2, 3 e 4, respectivamente). Em um dos componentes do esquema múltiplo, os reforços eram condicionados (fichas) e no outro os reforços eram incondicionados (comida). Os resultados dos Experimentos 2 e 3 indicaram maior resistência à mudança para os comportamentos mantidos pelos reforçadores condicionados. Por outro lado, os resultados do Experimento 4 indicaram maior resistência à mudança para os comportamentos mantidos pelos reforçadores incondicionados. Em relação ao resultado do Experimento 4, Vargo e Ringhdahl sugerem que a possibilidade de acesso à comida antes da sessão experimental, sem que respostas fossem exigidas para sua produção, pode ter afetado a relação entre os reforços condicionados e incondicionados produzindo, assim, resultados diferentes dos obtidos nos Experimentos 2 e 3 .

Os resultados obtidos nos estudos de Ahearn et al. (2003) e Vargo e Ringdahl (2015) são importantes na medida que demonstram generalidade das propostas da TMC acerca da relação S-S na determinação da resistência à mudança, independentemente do tipo de reforço avaliado. Os resultados desses estudos também têm grandes implicações para o tratamento de dependentes químicos, uma vez que o tratamento deve levar em consideração relações condicionais, incondicionais, tanto na resistência à mudança do comportamento de abuso quanto em sua possibilidade ressurgência após o tratamento. A seguir, serão apresentadas relações entre a área de estudo sobre resistência à mudança e ressurgência comportamental.

\section{Resistência à mudanca e ressurgência comportamental}

Ressurgência é a recorrência de um comportamento previamente reforçado, e depois extinto, quando os reforços para respostas alternativas são descontinuados ou têm seu valor reduzido (Epstein, 1983; Lattal et al., 2017). O procedimento típico para estudo do fenômeno envolve três fases: $\mathrm{Na}$ fase de Treino, uma dada RALV é reforçada; na fase de Eliminação (ou Reforçamento Alternativo), a RALV é extinta e uma RALT é reforçada. Tipicamente, na fase de Teste, a RALV continua em extinção e os reforços para a RALT também são descontinuados. A ressurgência é definida como um aumento na ocorrência da RALV na fase de Teste em relação à fase de Eliminação.

O procedimento para o estudo da ressurgência tem sido proposto como um modelo experimental de recaída (Podlesnik, Jimenez-Gomes \& Shahan, 2006; ver também, Bouton, 2014). Além de permitir uma análise da resistência à mudança de RALV na fase de Eliminação (note que reforçar uma RALT quando a RALV está em extinção é um procedimento de DRA), o procedimento permite avaliar, na fase de Teste, a resistência de RALT e a recorrência ou ressurgência de RALV.

Em uma série de experimentos com pombos e ratos, Podlesnik e Shahan $(2009,2010)$ demonstraram que a relação direta entre resistência à mudança e taxa e magnitude de reforços era também observada entre essas variáveis ambientais e a ressurgência comportamental. Assim, não apenas maior resistência, mas ressurgência de maior magnitude ocorria quando a RALV era mantida por maior taxa ou magnitude de reforços. Essa semelhança entre os determinantes da resistência à mudança e da ressurgência levaram Shahan e Sweeney (2011) a propor um modelo de ressurgência baseado na TMC. Em linhas gerais, o modelo afirma que a ressurgência é função direta da taxa (e magnitude) dos reforços na fase de Treino, assim como é uma função direta da taxa (e magnitude) dos reforços na fase de Eliminação, isto é, dos reforços alternativos. O papel dos reforços alternativos é duplo: ao mesmo tempo em que servem como operação perturbadora para a RALV, ocorrendo no 
mesmo contexto em que essa resposta era previamente reforçada, esses reforços fortalecem a relação S-S em vigor e, quando suspensos, aumentam a probabilidade de ressurgência ocorrer. Portanto, uma das predições do modelo é que, tudo o mais sendo igual, taxas maiores de reforços para a RALV produzirão maior ressurgência; da mesma forma, quando tudo mantido constante, taxas mais altas de reforços para a RALT (que são suspensos na fase de Teste), produzirão maior ressurgência.

Essas predições do modelo foram confirmadas em alguns estudos com não-humanos e com humanos (Cançado, Abreu-Rodrigues \& Aló, 2015; Pritchard et al., 2014; Sweeney \& Shahan, 2013), mas detalhes do modelo não têm sido confirmados de forma geral (e.g., Cançado \& Lattal, 2013; Craig \& Shahan, 2016; Fujimaki, Lattal, \& Sakagami, 2015), o que têm levado à revisões do modelo (Shahan \& Craig, 2017, por exemplo, propõem um modelo alternativo de ressurgência baseado na lei da igualação).

Independentemente de limites dos modelos teóricos, os resultados das pesquisas têm indicado que essa relação entre a taxa e a magnitude de reforços e a ressurgência comportamental parece ser confiável. Portanto, é algo a ser considerado na análise de comportamentos em contextos aplicados, e na elaboração e implementação de intervenções comportamentais (sobretudo aquelas que envolvem DRA). Idealmente, uma intervenção comportamental para um determinado comportamento problema têm como objetivo a redução na frequência deste e o aumento na frequência de respostas alternativas. Da mesma forma, deseja-se que, se houver falhas na integridade da intervenção comportamental, o comportamento alternativo persista e que ressurgência do comportamento problema (e.g., o abuso de drogas ou outro comportamento de relevância social ou clínica) não ocorra. Conhecer as variáveis que determinam a resistência à mudança e a ressurgência podem levar, portanto, ao desenvolvimento e à implementação de intervenções comportamentais mais eficazes (ver Fisher, Greer, Fuhrman, Saini, \& Simmons, 2018, para aplicação do quadro conceitual da TMC sobre a ressurgência comportamental em participantes com desordens comportamentais severas).

\section{Considerações finais}

Como proposto no início do presente artigo, foram descritos aspectos históricos, metodológicos e implicações para a Análise do Comportamento Aplicada relacionados ao desenvolvimento da TMC e da área de pesquisa sobre resistência do comportamento à mudança.

A breve revisão de literatura apresentada permite afirmar que a resistência do comportamento à mudança é um fenômeno complexo que pode ser afetado por diversas variáveis, tais como a taxa ou a magnitude dos reforços (principalmente) e diferentes exigências sobre a taxa de respostas na LB e atraso dos reforços (quando a taxa ou magnitude de reforços são igualadas). Atualmente, a resistência à mudança tem sido estudada em função da manipulação de outras variáveis como a dependência entre as respostas e os reforços (e.g., Cançado, Abreu-Rodrigues, Aló, Doughty \& Hauck, 2018), exigências sobre a força da resposta (e.g., Luiz, 2018; Pinkston \& Foss, 2018).

É certo que ainda existem muitas outras variáveis para serem manipuladas e a continuidade de produção de conhecimento nessa área de pesquisa fornecerá melhor conhecimento acerca do comportamento dos organismos em geral, e dos determinantes da resistência do comportamento à mudança, em particular (ver, sobretudo, Killeen \& Nevin, 2018 para uma revisão da TMC baseada nos Princípios Matemáticos do Reforço) e possibilitará uma análise mais precisa sobre a relação entre a resistência à mudança e outros fenômenos, como a ressurgência comportamental (ver, especialmente, Shahan \& Craig, 2017).

Além disso, a continuidade dos trabalhos nessa área de pesquisa, pode promover o desenvolvimento e a implementação de intervenções comportamentais mais efetivas. Intervenções que implementam DRA envolvem, em geral, a extinção de comportamentos alvo. Assim, podemos interpretar a intervenção como uma operação perturbadora sobre ocorrência de um comportamento alvo. O conhecimento sobre os determinantes da resistência à mudança (assim como da ressurgência, que ocorreria quando a intervenção, ou a operação perturbadora nessa analogia, fosse suspensa ou 
tivesse sua integridade comprometida) pode guiar trabalhos de maior qualidade e trazer mais benefícios para aqueles e aquelas que buscam o auxílio de analistas do comportamento. Como afirmou Nevin et al. (2017), ainda que a TMC tenha sérias limitações e necessite de revisões consistentes, o conhecimento produzido com base em seus pressupostos e previsões permite que o trabalho de analistas do comportamento seja guiado por evidências empíricas confiáveis. Essas mesmas evidências servirão para indicar a necessidade de alteração nos modelos teóricos que descrevem o fenômeno, assim como informarão o trabalho de analistas aplicados do comportamento nos mais diversos contextos.

\section{Referências}

Ahearn, W. H., Clark, K. M., Gardenier, N. C., Chung, B. I., \& Dube, W. V. (2003). Persistence of stereotypic behavior: examining the effects of external reinforcers. Journal of Applied Behavior Analysis, 36, 439-448. doi: 10.1901/ jaba.2003.36-439

Aló, R. M., Abreu-Rodrigues, J., Souza, A. S., \& Cançado, C. R. X. (2015). The persistence of fixed-ratio and differential-reinforcement-of-low-rate schedule performances. Revista Mexicana de Análisis de la Conducta, 41(1), 3-31. Disponível em: http://www.redalyc.org/ articulo.oa?id=59338802001

Aló, R. M., \& Costa, C. E. (no prelo). Força da resposta e resistência do comportamento à mudança: por que inisistimos tanto? In P. G. Soares, J. H. de Almeida, C.R.X. Cançado (Orgs.). Experimentos Clássicos em Análise do Comportamento, Vol.2. Brasília: Editora do Instituto Walden 4

Anger, D., \& Anger, K. (1976). Behavior changes during repeated eight-day extinctions. Journal of the Experimental Analysis of Behavior, 26(2), 181-190.doi: 10.1901/jeab.1976.26-181

Baum, W. M. (2012). Extinction as discrimination: The molar view. Behavioural processes, 90(1), 101-110. doi: 10.1016/j.beproc.2012.02.011

Bouton, M. E. (2014). Why behavior change us difficult to sustain. Preventive Medicine, 68, 29-36. doi: 10.1016/j.ypmed.2014.06.010
Cançado, C. R. X., Abreu-Rodrigues, J., \& Aló, R. M. (2015). Reinforcement rate and resurgence: A parametric analysis. Revista Mexicana de Análisis de la Conducta, 41, 84115. Disponível em: https://www.redalyc.org/ pdf/593/59341195006

Cançado, C. R., Abreu-Rodrigues, J., Aló, R. M., Hauck, F., \& Doughty, A. H. (2018). Responsereinforcer dependency and resistance to change. Journal of the Experimental Analysis of Behavior, 109(1), 176-193. doi: 10.1002/jeab.274

Cançado, C. R. X., \& Lattal, K. A. (2013). Response elimination, reinforcement rate and resurgence of operant behavior. Behavioural Processes, 100, 91-102. doi: 10.1016/j.beproc.2013.07.027

Cohen, S. L. (1996). Behavioral momentum of typing behavior in college students. Journal of Behavior Analysis and Therapy, 1, 36-51.

Cohen, S. L. (1998). Behavioral momentum: the effects of the temporal separation of rates of reinforcement. Journal of the Experimental Analysis of Behavior, 69(1), 29-47. doi: 10.1901/ jeab.1998.69-29

Costa, C. E., Cirino, S. D., Cançado, C. R. X., \& Soares, P. G. (2009). Polêmicas sobre história comportamental: identificação de seus efeitos e sua duração. Psicologia: Reflexão e Crítica, 22(3), 394-403. doi: 10.1590/S010279722009000300010

Craig, A. R., Nevin, J. A., \& Odum, A. L. (2014). Behavioral momentum and resistance to change. In McSweeney, F. K., \& Murphy, E. S. (Eds.), The Wiley-Blackwell handbook of operant and classical conditioning (pp. 249-274). Oxford, UK: Wiley-Blackwell.

Craig, A. R., \& Shahan, T. A. (2016). Behavioral momentum theory fails to account for the effects of reinforcement rate on resurgence. Journal of the Experimental Analysis of Behavior, 105(3), 375-392. doi:10.1002/jeab.207

Epstein, R. (1983). Resurgence of previously reinforced behavior during extinction. Behaviour Analysis Letters, 3, 391-397.

Fisher, W. W., Greer, B. D., Fuhrman, A. M., Saini, V., \& Simmons, C. A. (2018). Minimizing resurgence of destructive behavior using behavioral momentum theory. Journal of Applied Behavior Analysis, 51(4), 831-853. doi:10.1002/jaba.499 
Fujimaki, S., Lattal, K. A., \& Sakagami, T. (2015). A further look at reinforcement rate and resurgence. Mexican Journal of Behavior Analysis, 41(2), 116-136. Disponível em: https://www. redalyc.org/pdf/593/59341195007

Grace, R. C., Schwendiman, J. W., \& Nevin, J. A. (1998). Effects of unsignaled delay of reinforcement on preference and resistance to change. Journal of the Experimental Analysis of Behavior, 69(3), 247-261. doi: 10.1901/jeab.1998.69-247

Grimes, J. A., \& Shull, R. L. (2001). Response-independent milk delivery enhances persistence of pellet-reinforced lever pressing by rats. Journal of the Experimental Analysis of Behavior, 76(2), 179-194. doi: 10.1901/jeab.2001.76-179

Harper, D. N. (1996). Response-independent food delivery and behavioral resistance to change. Journal of the Experimental Analysis of Behavior, 65(3), 549-560. doi: 10.1901/jeab.1996.65-549

Harper, D. N., \& McLean, A. P. (1992). Resistance to change and the law of effect. Journal of the Experimental Analysis of Behavior, 57(3), 31737. doi: 10.1901/jeab.1992.57-317

Igaki, T., \& Sakagami, T. (2004). Resistance to change in goldfish. Behavioural Processes, 66(2), 139-152. doi: 10.1016/j.beproc.2004.01.009

Killeen, P. R., \& Nevin, J. A. (2018). The basis of behavioral momentum in the nonlinearity of strength. Journal of the experimental analysis of behavior, 109(1), 4-32. doi: 10.1002/jeab.304

Kuroda, T., Cançado, C. R., \& Podlesnik, C. A. (2016). Resistance to change and resurgence in humans engaging in a computer task. Behavioural processes, 125, 1-5. doi: 10.1016/j. beproc.2016.01.010Lattal, K. A. (1989). Contingencies on response rate and resistance to change. Learning and Motivation, 20(2), 191203. doi: 10.1016/0023-9690(89)90017-9

Lattal, K. A. (1989). Contingencies on response rate and resistance to change. Learning and Motivation, 20(2), 191-203.

Lattal, K. A., Cançado, C. R. X., Cook, J. E., Kincaid, S. L., Nighbor, T. D., \& Oliver, A. C. (2017). ON defining resurgence. Behavioural Processes, 141, 85-91. doi: 10.1016/j.beproc.2017.04.018

Luiz, A. (2018). Resistência do comportamento à mudança em função de diferentes forças exigidas para a ocorrência da resposta (Dissertação de Mestrado, Universidade Estadual de Londrina). Retirado em: http://www.bibliotecadigital.uel.br/document/?code $=$ vtls000220705 Mace, F. C., Lalli, J. S., Shea, M. C., Lalli, E. P., West, B. J., Roberts, M., \& Nevin, J. A. (1990). The momentum of human behavior in a natural setting. Journal of the Experimental Analysis of Behavior, 54(3), 163-172. doi: 10.1901/ jeab.1990.54-163

Mace, F. C., Lalli, J. S., West, B. J., Belfiore, P., \& Brown, D. K. (1988). Behavioral momentum in the treatmant of noncompliance. Journal of Applied Behavior Analysis, 2, 123-141. doi: 10.1901/jaba.1988.21-123

Mace, F. C., McComas, J. J., Mauro, B. C., Progar, P. R., Taylor, B., Ervin, R., \& Zangrillo, A. N. (2010). Differential reinforcement of alternative behavior increases resistance to extinction: Clinical demonstration, animal modeling, and clinical test of one solution. Journal of the Experimental Analysis of Behavior, 93(3), 349367. doi: 10.1901/jeab.2010.93-349

Mace, F. C., \& Nevin, J. A. (2017). Maintenance, Generalization, and Treatment Relapse: A Behavioral Momentum Analysis. Education and Treatment of Children, 40(1), 27-42. doi:10.1353/etc.2017.0001

Nevin, J. A. (1974). Response strength in multiple schedules. Journal of the Experimental Analysis of Behavior, 21(3), 389-408. doi: 10.1901/ jeab.1974.32-389

Nevin, J. (1996). The momentum of compliance. Journal of Applied Behavior Analysis, 29(4), 535-547. doi: 10.1901/jaba.1996.29-535

Nevin, J. A. (2015). Behavioral momentum: A scientific metaphor. Vineyard Haven: The Tisbury Printer.

Nevin, J. A., Craig, A. R., Cunningham, P. J., Podlesnik, C. A., Shahan, T. A., \& Sweeney, M. M. (2017). Quantitative models of persistence and relapse from the perspective of Behavioral Momentum Theory: Fits and misfits. Behavioural Processes, 141, 92-99. doi: 10.1016/j.beproc.2017.04.016

Nevin, J. a, \& Grace, R. C. (2000). Behavioral momentum and the law of effect. The Behavioral and Brain Sciences, 23(1), 73-90; discussion 90130. doi: 10.1017/S0140525X00002405 
Nevin, J. A., Mandell, C., \& Atak, J. R. (1983). The analysis of behavioral momentum. Journal of the Experimental Analysis of Behavior, 39, 4959. doi: 10.1901/jeab.1983.39-49

Nevin, J. A., \& Shahan, T. A. (2011). Behavioral Momentum Theory: Equations and applications. Journal of Applied Behavior Analysis, 44(4), 877-895. doi: 10.1901/jaba.2011.44-877

Nevin, J. A., Tota, M. E., Torquato, R. D., \& Shull, R. L. (1990). Alternative reinforcement increases resistance to change: Pavlovian or operant contingencies? Journal of the Experimental Analysis of Behavior, 53(3), 359-379. doi: 10.1901/ jeab.1990.53-359

Nevin, J. A., \& Wacker, D. P. (2013). Response strength and persistence. In G. J. Madden, W. V. Dube, T. D. Hackenberg, G. P. Hanley, \& K. A. Lattal (Eds.), APA handbook of behavior analysis, Vol. 2. Translating principles into practice (pp. 109-128). Washington, DC, US: American Psychological Association. doi: 10.1037/13938-005

Pinkston, J. W., \& Foss, E. K. (2018). The role of response force on the persistence and structure of behavior during extinction. Journal of the Experimental Analysis of Behavior, 109(1), 194-209. doi:10.1002/jeab.306

Podlesnik, C. A., Bai, J. Y. H., \& Elliffe, D. (2012). Resistance to extinction and relapse in combined stimulus contexts. Journal of the Experimental Analysis of Behavior, 98(2), 169189. doi:10.1901/jeab.2012.98-169

Podlesnik, C. A., Jimenez-Gomez, C., \& Shahan, T. A. (2006). Resurgence of alcohol seeking produced by discontinuing non-drug reinforcement as an animal model of drug relapse. Behavioural Pharmacology, 17(4), 369-374. doi: 10.1097/01.fbp.0000224385.09486.ba

Podlesnik, C. A., \& Shahan, T. A. (2008). Responsereinforcer relations and resistance to change. Behavioural Processes, 77(1), 109-125. doi: 10.1016/j.beproc.2007.07.002

Podlesnik, C. A., \& Shahan, T. A. (2009). Behavioral momentum and relapse of extinguished operant responding. Learning \& Behavior, 37(4), 357-364. doi: 10.3758/LB.37.4.357

Podlesnik, C. A., \& Shahan, T. A. (2010). Extinction, relapse, and behavioral momentum. Behavioural Processes, 84(1), 400-411. doi: 10.1016/j.beproc.2010.02.001

Ponce, G. D. (2014). Efeito do custo da resposta sobre a resistência do comportamento à mudança. (Dissertação de Mestrado, Universidade Estadual de Londrina). Retirado em: http://www.bibliotecadigital.uel.br/ document $/$ ?code $=$ vtls000200913

Pritchard, D., Hoerger, M., \& Mace. F. C., Penney, H., \& Harris, B. (2014). Clinical translation of animal models of treatment relapse. Journal of the Experimental Analysis of Behavior, 101(3), 442-449. doi: 10.1002/jeab.87

Reynolds, G. S. (1961). Behavioral contrast. Journal of the Experimental Analysis of Behavior, 4(1), $57-71$

Sampaio, A. A. S., Azevedo, F. H. B. D., Cardoso, L. R. D., Lima, C. D., Pereira, M. B. R., \& Andery, M. A. P. A. (2008). Uma introdução aos deli-neamentos experimentais de sujeito único. Interação em Psicologia, 12(1), 151-164. doi: 10.5380/psi.v12i1.9537

Santos, C. V. (2005). Momento comportamental. Em J. Abreu-Rodrigues \& M. R. Ribeiro (Orgs.), Análise do Comportamento: Pesquisa, Teoria e Aplicação (pp. 63-80). Porto Alegre: Artmed.

Saini, V., Fisher, W. W., \& Pisman, M. D. (2017). Persistence during and resurgence following noncontingent reinforcement implemented with and without extinction. Journal of Applied Behavior Analysis, 50(2), 377-392. doi:10.1002/ jaba.380

Shahan, T. A., \& Craig, A. R. (2017). Resurgence as choice. Behavioural Processes, 141, 100-127. doi: 10.1016/j.beproc.2016.10.006

Shahan, T. A., \& Burke, K. A. (2004). Ethanolmaintained responding of rats is more resistant to change in a context with added non-drug reinforcement. Behavioural Pharmacology, 15(4), 279-285. doi: 10.1097/01. fbp.0000135706.93950.1 a

Shahan, T. A., \& Sweeney, M. M. (2011). A model of resurgence based on behavioral momentum theory. Journal of the Experimental Analysis of Behavior, 95(1), 91-108. doi: 10.1901/ jeab.2011.95-91

Sidman, M. (1976). Táticas da pesquisa científica: Avaliação dos dados experimentais na psicologia (M. E. Paiva, Trad.). São Paulo, SP: Brasiliense. 
(Original publicado em 1960)

Skinner, B. F. (1938). The behavior of organisms. Englewood Cliffs, NJ: Prentice Hall.

Sweeney, M. M., \& Shahan, T. A (2013). Effects of high, low, and thinning rates of alternative reinforcement on response elimination and resurgence. Journal of the Experimental Analysis of Behavior, 100(1), 102-116. doi: 10.1002/ jeab.26

Vargo, K. K., \& Ringdahl, J. E. (2015). An evaluation of resistance to change with unconditioned and conditioned reinforcers. Journal of Applied Behavior Analysis, 48(3), 643-662. doi:10.1002/ jaba.226

Vollmer, T. R., Iwata, B. A., Zarcone, J. R., Smith, R. G., \& Mazaleski, J. L. (1993). The role of attention in the treatment of attention-maintained self-injurious behavior: noncontingent reinforcement and differential reinforcement of other behavior. Journal of Applied Behavior Analysis, 26(1), 9-21. doi:10.1901/jaba.1993.26-9

\section{Informações do Artigo}

Histórico do artigo:

Submetido em: 31/08/2018

Primeira decisão editorial: 10/12/2018

Versão definitiva aceita em: 05/04/2019

Editor Associado: Denis Roberto Zamignani 